\title{
Designing an Ultra-Negative Dispersion Photonic Crystal Fiber with Square-Lattice Geometry
}

\author{
Partha Sona Maji and Partha Roy Chaudhuri \\ Department of Physics and Meteorology, Indian Institute of Technology, Kharagpur 721302, India \\ Correspondence should be addressed to Partha Sona Maji; parthamaji.1984@gmail.com
}

Received 8 February 2014; Accepted 6 March 2014; Published 1 April 2014

Academic Editors: Y. S. Kivshar and S. Shi

Copyright (c) 2014 P. S. Maji and P. Roy Chaudhuri. This is an open access article distributed under the Creative Commons Attribution License, which permits unrestricted use, distribution, and reproduction in any medium, provided the original work is properly cited.

\begin{abstract}
We have theoretically investigated the dispersion characteristics of dual-core PCF, based on square-lattice geometry by varying different parameters. The fiber exhibits a very large negative dispersion because of rapid slope change of the refractive indices at the coupling wavelength between the inner core and outer core. The dependence of different geometrical parameters, namely, hole-tohole spacing $(\Lambda)$ and different air-hole diameter $(d)$, was investigated in detail. By proper adjustment of the available parameters, a high negative dispersion value of $-47,500 \mathrm{ps} / \mathrm{nm} / \mathrm{km}$ has been achieved around the wavelength of $1550 \mathrm{~nm}$. Our proposed fiber will be an excellent device for dispersion compensation in long-haul data transmission as being thousand times more than the available DCFs.
\end{abstract}

\section{Introduction}

Photonic crystal fibers (PCFs) [1, 2] or Holey Optical Fibers offered a tremendous variety of possible geometries utilizing the shape, size, and positioning of air-holes in the microstructured cladding. The air-hole diameter $(d)$ and hole-to-hole spacing $(\Lambda)$ not only control the dispersion properties, but also the transmission and the nonlinear properties of the fiber as well. Achieving very high negative values of dispersion around the communication band has been the target for a long time [3-15].

The principle behind having a very large negative dispersion in these Dispersion Compensating Fibers (DCFs) being the coupling between two spatially separated asymmetric concentric cores which support two leaky modes: inner mode and outer mode. By proper design, mode matching can take place between these two modes at the desired wavelength. A few analyses have been performed to realize high negative dispersion with triangular lattice PCF [8-15]. In this work we have studied rigorously towards achieving high negative dispersion value with regular square lattice. Square-latticebased PCF is superior to triangular-lattice PCF for certain properties $[16,17]$. Square-lattice PCF shows wider range of single mode operation with the same $d / \Lambda$ value compared to the triangular one [16]. The effective area of square-lattice PCF is higher than triangular one, making the former better for high power management [17]. Square-lattice PCF can better compensate the inline dispersion around the $1550 \mathrm{~nm}$ wavelength than the triangular-lattice PCF [17].

In recent times, a square-lattice PCF preform has been realized with a standard fabrication process, stack and draw, in order to study the localization and control of high frequency sound by introducing two solid defects in the periodic distribution of air-holes [18]. Thus the technological feasibility of the square-lattice PCFs has been demonstrated, since the final PCFs can be obtained by drawing the intermediate prepared preforms [18]. In another example, experimental study of negative refraction has been studied with squarelattice photonic crystal [19]. So, square-lattice PCF can be experimentally realized like that of the usual triangular-lattice PCF.

\section{Geometry of the Structure and Analysis Method}

Cross-sectional geometry of the proposed/studied fiber has been shown in Figure 1. It is well known that a triangular 


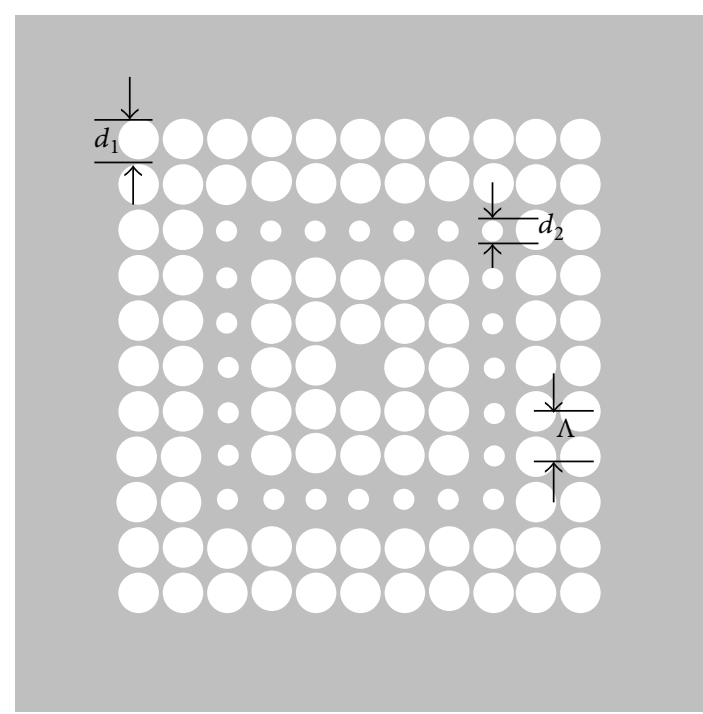

FIgURE 1: Cross-section of the proposed/studied fiber. The air-hole diameter of the third air-hole ring is reduced to create the outer core, thereby creating the dual-core structure.

lattice PCF is usually described by air-hole diameter $d$ and hole-to-hole distance (also called pitch) $\Lambda$. Now, we use $\Lambda$ as the hole-to-hole spacing both in horizontal and vertical directions in the square-lattice PCF geometry with $d_{1}$ as the diameter of the bigger air- holes. The central air-hole is missing, making it the inner core. The inner cladding is formed by the first two air-hole rings with air-hole diameter $d_{1}$. The diameter of the air-holes for the 3rd air-hole ring is reduced, thereby increasing the local refractive index of the ring, making the ring as the outer core. The diameter of the air-holes in this outer ring is represented as $d_{2}$. The rings of holes beyond the third rings form the outer cladding with air-hole diameter $d_{1}$. The background of the fiber is taken to be silica whose refractive index has been considered through Sellmeier's equation

$$
n^{2}(\lambda)=A+\frac{B_{1} \lambda^{2}}{\lambda^{2}-\lambda_{1}{ }^{2}}+\frac{B_{2} \lambda^{2}}{\lambda^{2}-\lambda_{2}{ }^{2}}+\frac{B_{3} \lambda^{2}}{\lambda^{2}-\lambda_{3}^{2}}
$$

with $B_{1}=0.696166300, B_{2}=0.407942600, B_{3}=$ $0.897479400, \lambda_{1}=0.0684043 \mu \mathrm{m}, \lambda_{2}=0.1162414 \mu \mathrm{m}$, and $\lambda_{3}=9.896161 \mu \mathrm{m}[20]$.

We solve the guided modes of the present fiber by the CUDOS MOF Utilities [21] that simulate PCFs using the multipole method $[22,23]$. We have calculated the dispersion parameter using

$$
D=-\frac{\lambda}{c} \frac{d^{2} \operatorname{Re}\left[n_{\mathrm{eff}}\right]}{d \lambda^{2}}
$$

with $\operatorname{Re}\left(n_{\text {eff }}\right)$ being the real part of the effective indices obtained from simulations and $c$ being the speed of light in vacuum.

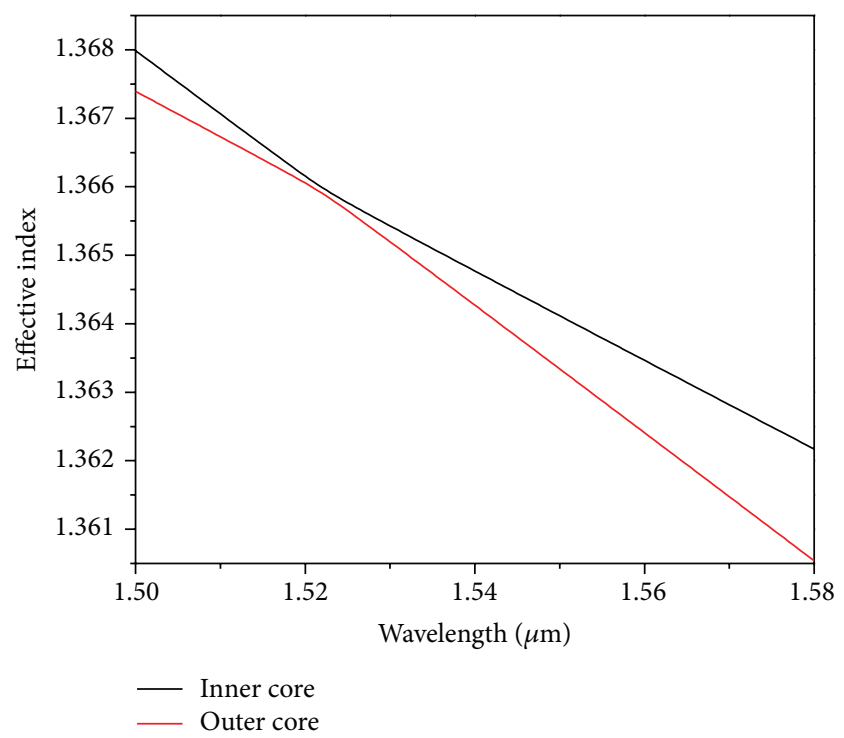

FIGURE 2: Variation of real part of the effective indices for both the cores (inner core-black line and outer core red-line) of PCFs with $\Lambda=1.40 \mu \mathrm{m}, d_{1}=1.12 \mu \mathrm{m}$, and $d_{2}=0.56 \mu \mathrm{m}$.

The confinement loss for the structures has been calculated through

$$
L=\frac{2 \pi}{\lambda} \frac{20}{\ln (10)} 10^{6} \operatorname{Im}\left(n_{\text {eff }}\right) \frac{d B}{m},
$$

where $\operatorname{Im}\left(n_{\text {eff }}\right)$ is the imaginary part of the effective indices (obtained from the simulations) with $\lambda$ is in micrometer.

\section{Dispersion Analysis of the Structure}

We started our dispersion analysis with $\Lambda=1.40 \mu \mathrm{m}$ and $d_{1} / \Lambda=0.8$ and $d_{2} / d_{1}=0.5$. A very high negative dispersion of $-21,700 \mathrm{ps} / \mathrm{nm} / \mathrm{km}$ around the wavelength of $1522 \mathrm{~nm}$ was observed as shown in Figure 4. The corresponding variations of effective index have been presented in Figure 2 which shows a distinctive change of slope at the coupling wavelength. The variation of the indices for both the inner and outer cores is presented in Figure 2. The cross-off between the two cores (inner core and outer core) can be better viewed from Figure 3 which represents the imaginary part of the refractive indices $\left(\operatorname{Im}\left(n_{\text {eff }}\right)\right)$ of the two cores. The two curves meet at the coupling wavelength of $1522 \mathrm{~nm}$. After the coupling, most of the power in the inner core goes to the outer core. This principle can be used for suppressing spontaneous emission of certain wavelengths.

The dependence of dispersion upon the geometrical parameters $\left(d_{1}, d_{2}\right.$, and $\left.\Lambda\right)$ has been presented in Figures 5-7. The dependence of the variation of bigger air-holes $\left(d_{1}\right)$ upon dispersion has been presented in Figure 5. For this purpose we have kept $\Lambda=1.40 \mu \mathrm{m}$, keeping $d_{2} / d_{1}=0.5$. From the figure it is clearly visible that the absolute values of the biggest negative dispersion increase as $d_{1}$ increases (air filling rate increases), the corresponding coupling wavelength is red-shifted, and the absolute values of the dispersion 


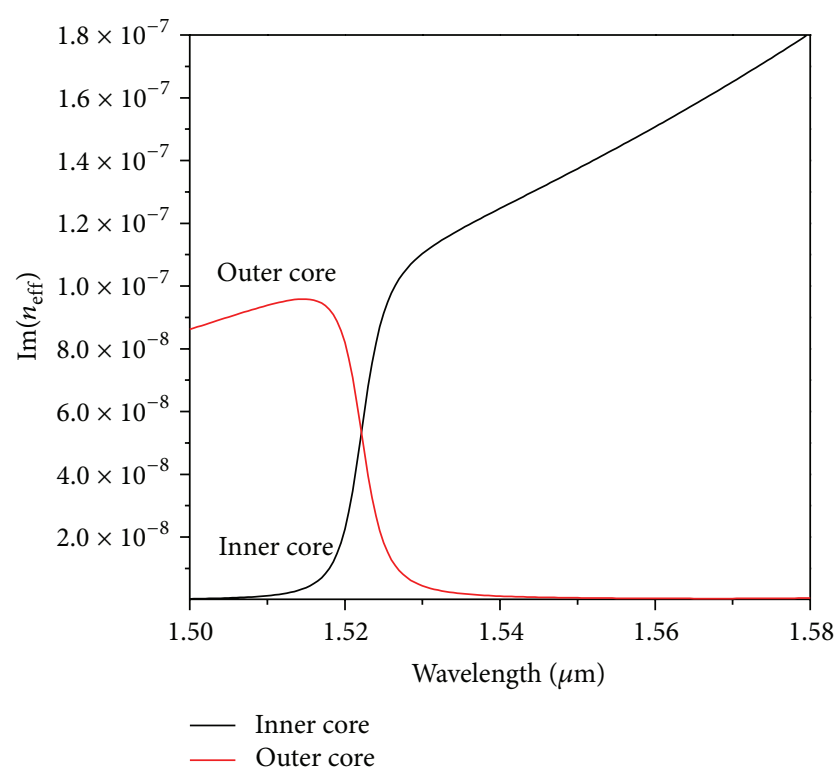

FIGURE 3: $\operatorname{Im}\left(n_{\text {eff }}\right)$ variation for the two cores of the PCFs with $\Lambda=$ $1.4 \mu \mathrm{m}, d_{1}=1.12 \mu \mathrm{m}$, and $d_{2}=0.56 \mu \mathrm{m}$.

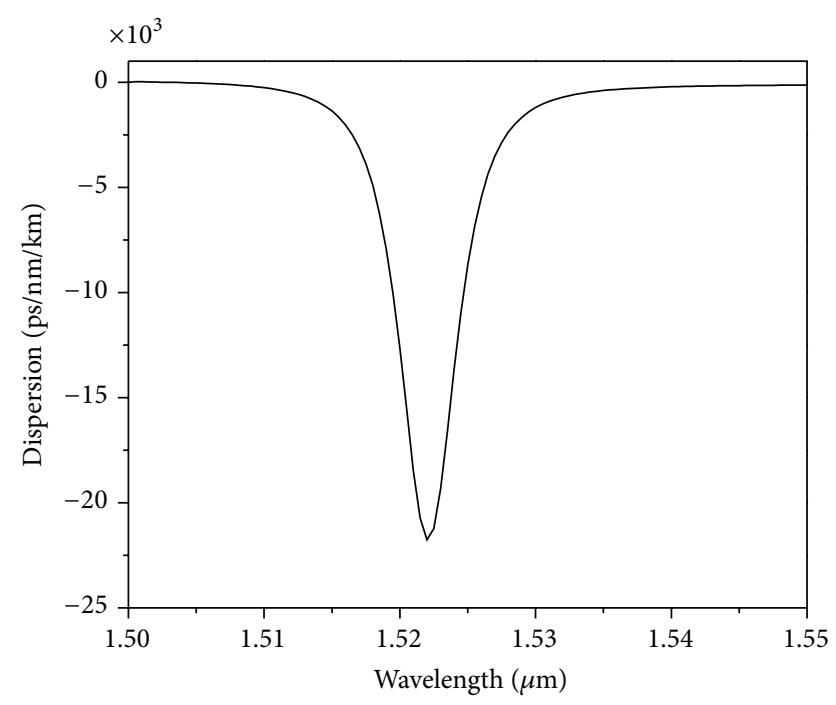

Figure 4: Dispersion curve for PCFs with $\Lambda=1.40 \mu \mathrm{m}, d_{1}=1.12 \mu \mathrm{m}$ and $d_{2}=0.56 \mu \mathrm{m}$.

slope increase, but the value of full width at half maximum (FWHM) decreases. From the figure it can be easily observed that with an increase of negative dispersion the corresponding FWHM decreases making the product of bandwidth and peak dispersion almost constant. The dispersion curves of PCFs for different $d_{2}$ have been presented in Figure 6 with $\Lambda=1.40 \mu \mathrm{m}$ and $d_{1}=1.12 \mu \mathrm{m}$. The figure clearly represents that values of the biggest negative dispersion decrease as $d_{2}$ increases (air filling rate of the outer core increases), the corresponding coupling wavelength is red-shifted, and the absolute values of the dispersion slope decrease. Here with the increases of outer air-hole diameter the peak dispersion decreases, but the corresponding FWHM increases keeping

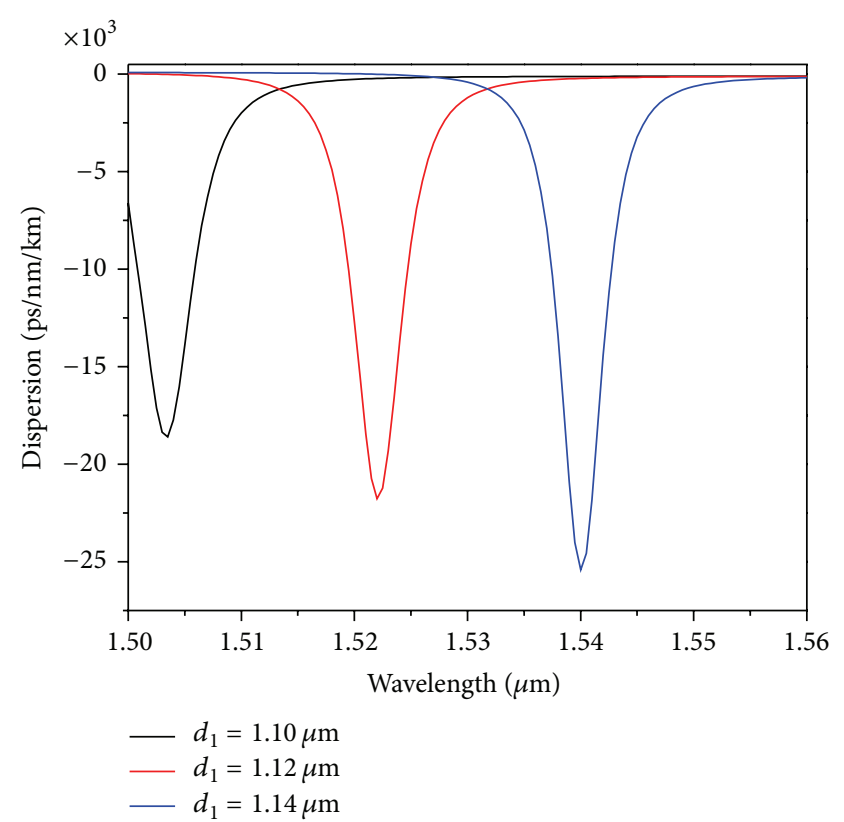

FIGURE 5: Variation of dispersion for $\Lambda=1.40 \mu \mathrm{m}$ for $d_{2} / d_{1}=0.5$ for different values of $d_{1}(1.10 \mu \mathrm{m}, 1.12 \mu \mathrm{m}$, and $1.14 \mu \mathrm{m})$.

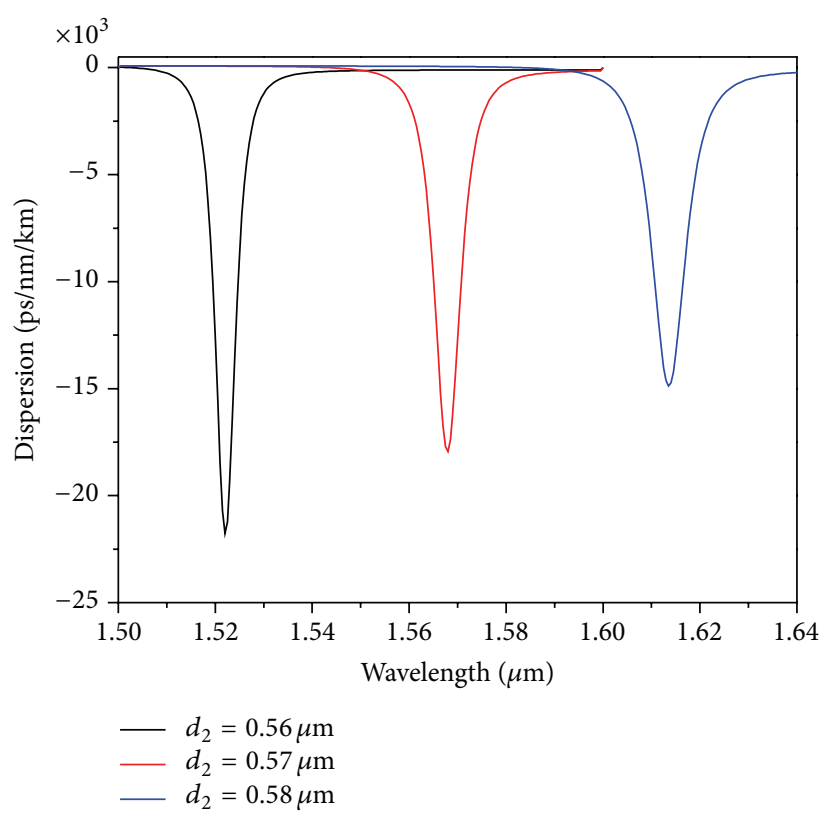

FIGURE 6: Variation of $D$ for $\Lambda=1.40 \mu \mathrm{m}$ for different values of $d_{2}$ $(0.55 \mu \mathrm{m}, 0.56 \mu \mathrm{m}$, and $0.57 \mu \mathrm{m})$ with $d_{1}=1.12 \mu \mathrm{m}$.

the product of bandwidth and peak dispersion almost constant. The dependence of hole-to-hole distance $(\Lambda)$ upon dispersion has been presented in Figure 7. For this purpose we have considered two different PCFs with $d_{1} / \Lambda=0.8$ and $d_{2} / d_{1}=0.5$. Figure 7 clearly represents that the absolute values of the biggest negative dispersion reduces appreciably as $\Lambda$ increases and the analogous wavelength is red-shifted and the absolute values of the dispersion slope decrease significantly. 


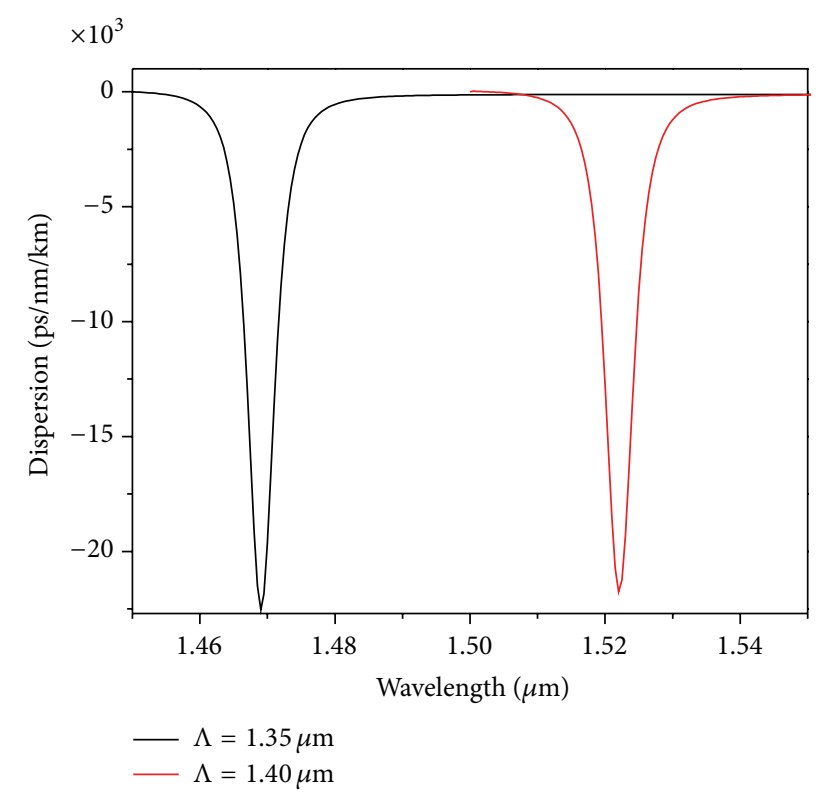

Figure 7: Variation of $D$ for different values of $\Lambda$ with $d_{2} / d_{1}=0.5$ and $d_{1} / \Lambda=0.8$.

In this section, we will study the fabrication tolerance of the dual core S-PCF for designing ultra-low dispersion at the required wavelength. For this purpose, we have considered the S-PCF as demonstrated in Figure 4 with $\Lambda=1.40 \mu \mathrm{m}, d_{1}=1.12 \mu \mathrm{m}$, and $d_{2}=0.56 \mu \mathrm{m}$. The tolerances have been considered for four values including the original values. For this case, we have changed the values of the available parameters $\left(\Lambda, d_{1}\right.$, and $\left.d_{2}\right)$ with a change from $-2 \%$ to $+2 \%$ in a step of $1 \%$. The variation of the dispersion for percentage change of $\Lambda, d_{1}$, and $d_{2}$ has been demonstrated in Figures 8,9 , and 10 , respectively. The figures reveal that for a decrease of $\Lambda$, peak dispersion increases towards a smaller wavelength (Figure 8(a)), while peak dispersion increases with an increase of " $d_{1}$ " towards a smaller wavelength (Figure 9(a)) with dispersion increase with a decrease of " $d_{2}$ " (Figure 10(a)). The figures (Figures $8(\mathrm{~b}), 9(\mathrm{~b})$, and 10 (b)) reveal that peak dispersion wavelength variation is almost linear with a change of parameters. However, the same cannot be concluded for peak dispersion variation (Figures $8(\mathrm{a}), 9(\mathrm{a})$, and 10(a)) with parameters and the variation is close to linear but deviates from linear relationship. One of the important information that can be concluded from Figures $8-10$ is that the peak dispersion wavelength value is most sensitive to the change of smaller air-hole diameter $\left(d_{2}\right)$, while the effect of change of hole-to-hole distance $(\Lambda)$ is the least. Another interesting fact can be observed; that is, peak dispersion is most sensitive to the change of bigger air-hole diameter $\left(d_{1}\right)$, while hole-to-hole distance $(\Lambda)$ effects the least change. These engineering aspects of effect of change of parameters by a certain percentage towards different peak dispersion and corresponding wavelength will be of great help towards possible fabrication of a dual-core SPCF for narrowband ultra-negative dispersion.

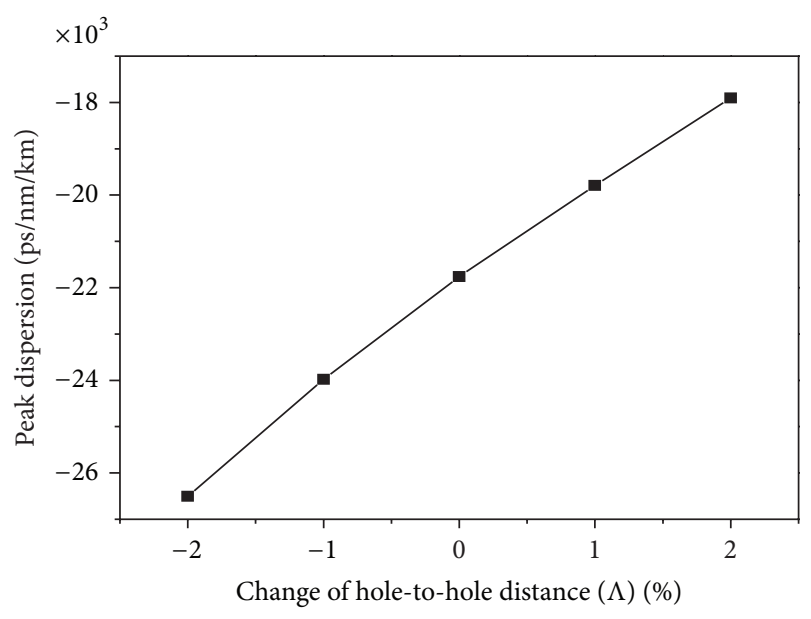

(a)

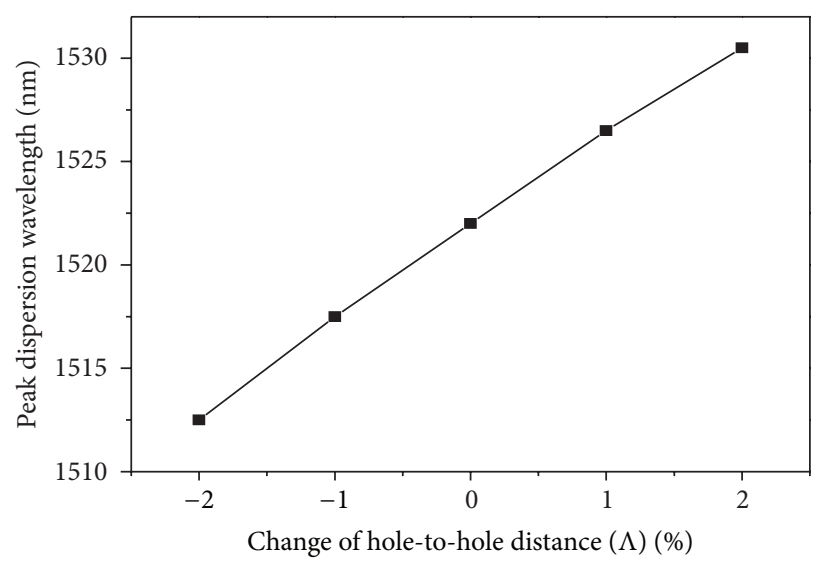

(b)

FIGURE 8: Variation of peak dispersion (a) and peak dispersion wavelength (b) for percentage change of hole-to-hole distance $(\Lambda)$.

\section{Ultra-Negative Dispersion Compensating Dual-Core PCF}

Based upon the above studies we changed the available parameters and we could achieve a very high negative dispersion of $-47,500 \mathrm{ps} / \mathrm{nm} / \mathrm{km}$ around $1550 \mathrm{~nm}$ with $\Lambda=$ $1.4 \mu \mathrm{m}, d_{1}=1.196 \mu \mathrm{m}$, and $d_{2}=0.59 \mu \mathrm{m}$ as shown in Figure 11 . This value of negative dispersion is the highest to the best of our knowledge as the value is more than twice than the earlier reported value with regular triangular-lattice PCF [7-13] that can be drawn with regular stack and draw method. This optimized designed PCF will be very helpful in compensating the inline dispersion caused by SMF-28. For example, the dispersion caused by existing SMF-28 is calculated through (4) with a zero dispersion wavelength $\lambda_{0}$ of $1311.5 \mathrm{~nm}$ and a zero dispersion slope $S_{0}$ of $0.092 \mathrm{ps} \mathrm{nm}^{-2} \mathrm{~km}^{-1}$ and is given by [24]

$$
D(\lambda)=\frac{S_{0}}{4}\left[\lambda-\frac{\lambda_{0}^{4}}{\lambda^{3}}\right]
$$




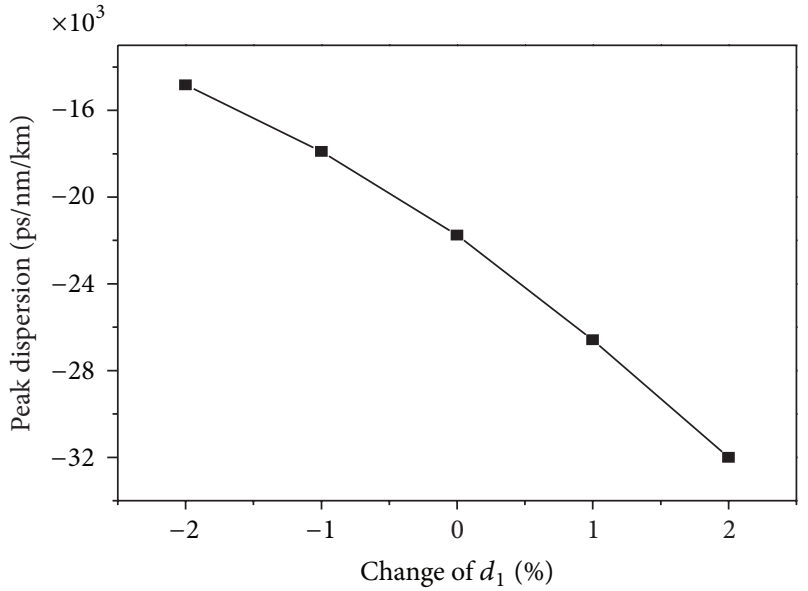

(a)

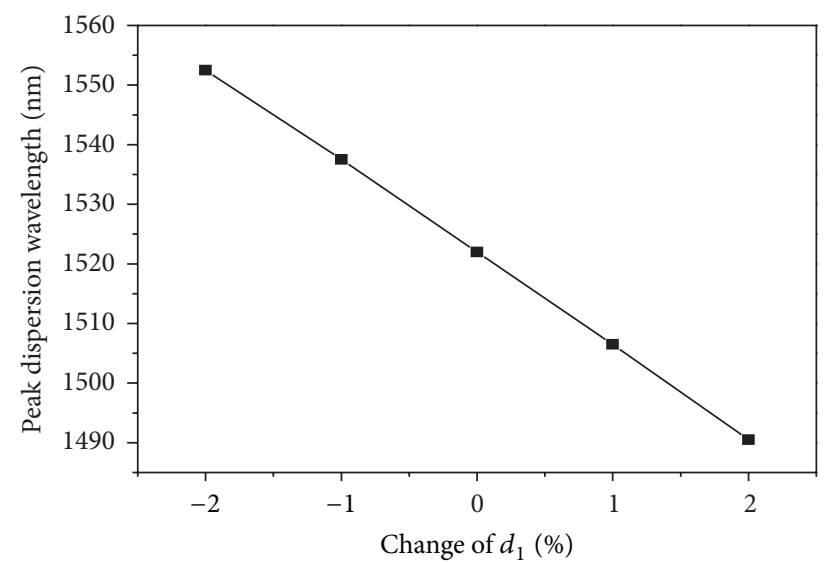

(b)

FIGURE 9: Variation of peak dispersion (a) and peak dispersion wavelength (b) for percentage change of bigger air-hole diameter $\left(d_{1}\right)$.

At the wavelength of $1550 \mathrm{~nm}$ the dispersion caused by the existing fiber is $17.378 \mathrm{ps} / \mathrm{nm} / \mathrm{km}$. So with a negative dispersion of $-47,500 \mathrm{ps} / \mathrm{nm} / \mathrm{km}$ of the optimized design, we can compensate the dispersion caused by $27.3 \mathrm{~km}$ of the existing inline optical fiber with only 10 meters of our optimized fiber. The confinement loss of the above PCF was calculated with (3) and the value was found to be $0.089 \mathrm{~dB} / \mathrm{m}$ at the wavelength of $1550 \mathrm{~nm}$. To compensate the dispersion caused by $27.1 \mathrm{~km}$ of SMF- 28 as mentioned above, the total propagation losses will be $0.89 \mathrm{~dB}$ for a propagation of 10 meters. So with this small length of the fiber not only the inline dispersion will be compensated but also the propagation losses caused by the fiber can also be reduced significantly. Similar requirements of short length fiber based devices can also be accomplished by the present kind of devices. Effective area is another important parameter for long-haul communications. The effective area variation for the optimized PCF has been presented in Figure 12 for the entire C-band of wavelength. We have calculated the splice loss between the SMF-28 and the present fiber around $1550 \mathrm{~nm}$ of wavelength and the value was found to be $2.2 \mathrm{~dB}$.

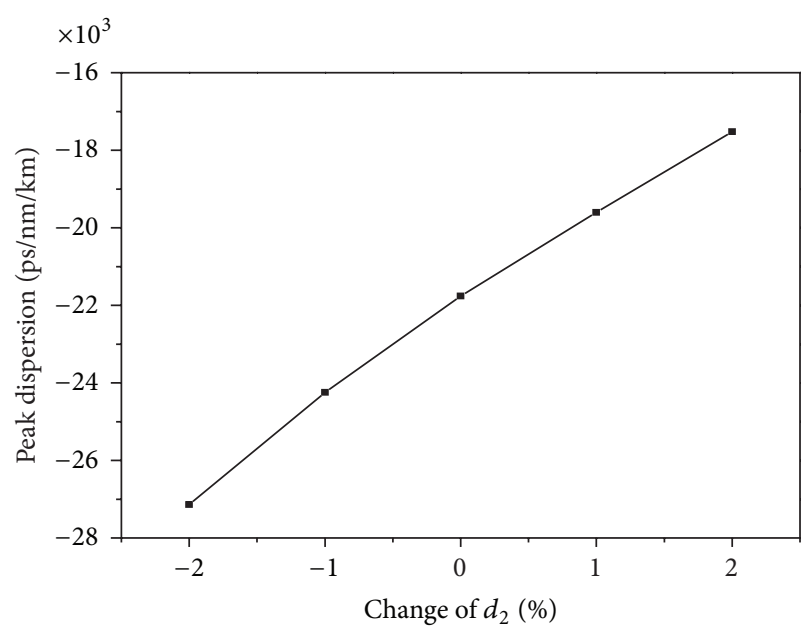

(a)

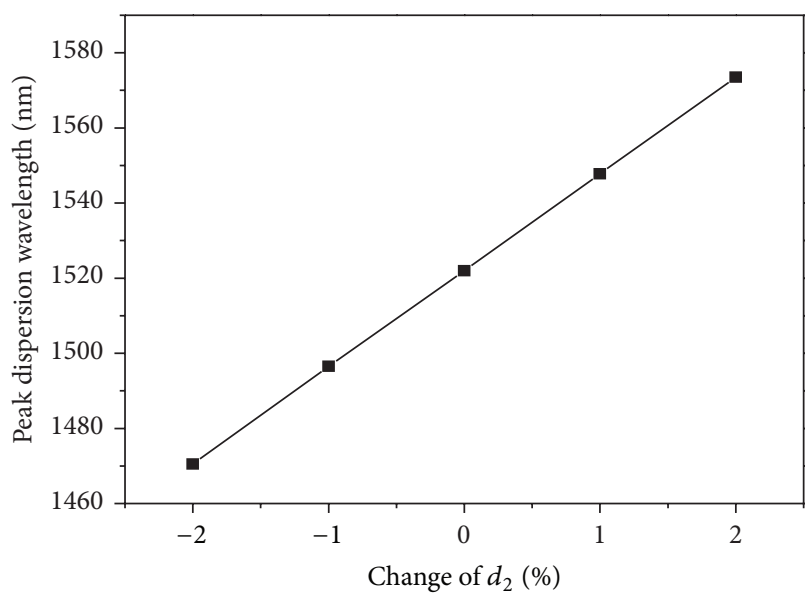

(b)

FIgURE 10: Variation of peak dispersion (a) and peak dispersion wavelength (b) for percentage change of smaller air-hole diameter $\left(d_{2}\right)$.

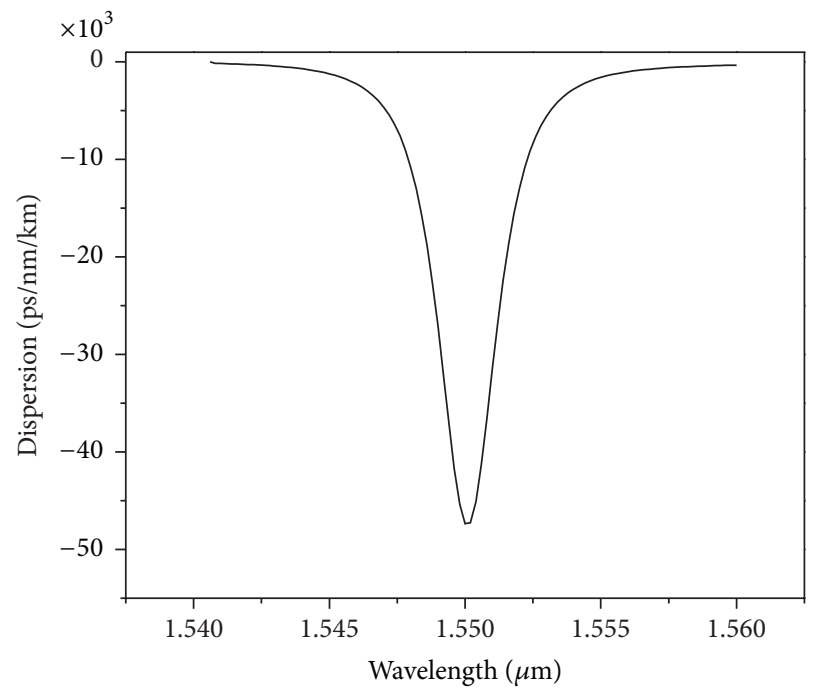

FIGURE 11: Optimized dispersion value of $-47,500 \mathrm{ps} / \mathrm{nm} / \mathrm{km}$ achieved with $\Lambda=1.40 \mu \mathrm{m}$ with $d_{1}=1.196 \mu \mathrm{m}$ and $d_{2}=0.59 \mu \mathrm{m}$. 


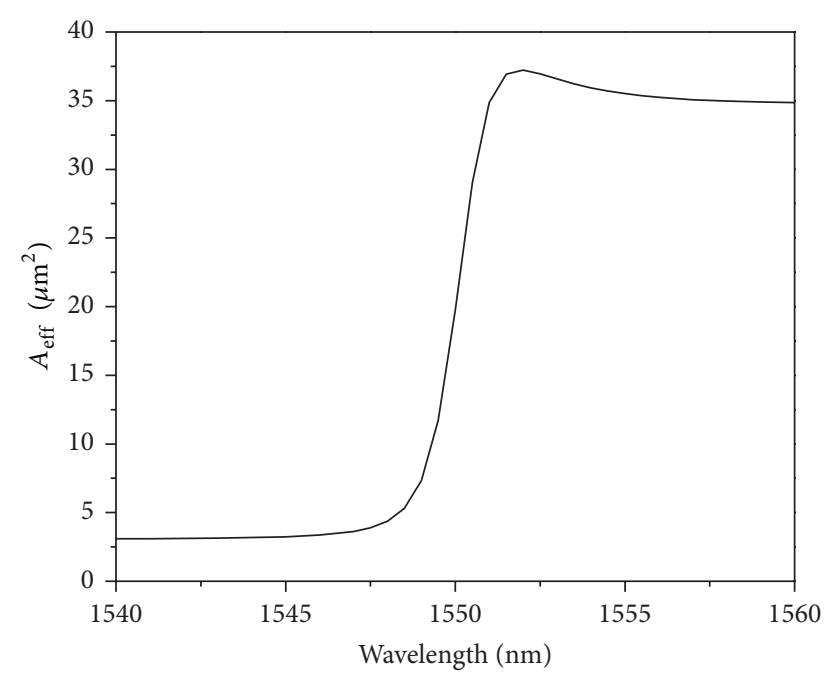

FIGURE 12: Effective area variation of the optimized PCF.

The relatively higher loss can be attributed to the mismatch between the differences between the modal effective areas of the two fibers.

\section{Conclusions}

In this paper we have theoretically investigated chromatic dispersion compensation property exhibited by square-lattice geometry of the PCFs based on pure silica. We have extensively studied the effect of different geometrical parameters upon dispersion towards achieving ultra-negative dispersion. We have shown that with an increase of bigger air-hole diameter, the peak dispersion is red-shifted with higher negative dispersion at the cost of narrower FWHM while an increase of smaller air-hole diameter in the outer core again red-shifted the coupling wavelength but with smaller values of negative dispersion. Changing hole-to-hole distance has the effect of red-shifting the coupling wavelength with smaller values of negative dispersion. Based upon the above findings we could achieve an ultra-negative dispersion of $-47,000 \mathrm{ps} / \mathrm{nm} / \mathrm{km}$ around $1550 \mathrm{~nm}$ of wavelength by properly changing the parameters. Our designed fiber will be very useful for dispersion compensation in long-haul data transmission some thousand times more than the available DCFs. The basic principle of power transform from inner core to the outer core after the coupling can be applied for suppressing spontaneous emission after a particular wavelength.

\section{Conflict of Interests}

The authors would like to declare that there is no direct financial relation with any commercial identity mentioned in their work that might lead to a conflict of interests.

\section{Acknowledgments}

The authors would like to thank Dr. Boris Kuhlmey, University of Sydney, Australia, for providing valuable suggestions in understanding the software for designing and studying the properties of different structures. The authors acknowledge sincerely the Defence Research and Development Organization, Government of India, and CRF of IIT Kharagpur for the financial support to carry out this research.

\section{References}

[1] J. Broeng, D. Mogilevstev, S. E. Barkou, and A. Bjarklev, "Photonic crystal fibers: a new class of optical waveguides," Optical Fiber Technology, vol. 5, no. 3, pp. 305-330, 1999.

[2] J. C. Knight, "Photonic crystal fibers," Nature, vol. 424, no. 6950, pp. 847-851, 2003.

[3] L. P. Shen, W.-P. Huang, G. X. Chen, and S. S. Jian, "Design and optimization of photonic crystal fibers for broad-band dispersion compensation," IEEE Photonics Technology Letters, vol. 15, no. 4, pp. 540-542, 2003.

[4] A. Huttunen and P. Törmä, "Optimization of dual-core and microstructure fiber geometries for dispersion compensation and large mode area," Optics Express, vol. 13, no. 2, pp. 627-635, 2005.

[5] G. Prabhakar, A. Peer, V. Rastogi, and A. Kumar, "Largeeffective-area dispersion-compensating fiber design based on dual-core microstructure," Applied Optics, vol. 52, no. 19, pp. 4505-4509, 2013.

[6] G. Ouyang, Y. Xu, and A. Yariv, "Theoretical study on dispersion compensation in air-core Bragg fibers," Optics Express, vol. 10, no. 17, pp. 899-908, 2002.

[7] T. D. Engeness, M. Ibanescu, S. G. Johnson et al., "Dispersion tailoring and compensation by modal interactions in Omni Guide fibers," Optics Express, vol. 11, no. 10, pp. 1175-1196, 2003.

[8] F. Poli, A. Cucinotta, M. Fuochi, S. Selleri, and L. Vincetti, "Characterization of microstructured optical fibers for wideband dispersion compensation," Journal of the Optical Society of America A: Optics and Image Science, and Vision, vol. 20, no. 10, pp. 1958-1962, 2003.

[9] L.-P. Shen, W.-P. Huang, and S.-S. Jian, "Design of photonic crystal fibers for dispersion-related applications," Journal of Lightwave Technology, vol. 21, no. 7, pp. 1644-1651, 2003.

[10] B. Zsigri, J. Lægsgaard, and A. Bjarklev, "A novel photonic crystal fibre design for dispersion compensation," Journal of Optics A: Pure and Applied Optics, vol. 6, no. 7, pp. 717-720, 2004.

[11] Y. Ni, L. Zhang, L. An, J. Peng, and C. Fan, "Dual-core photonic crystal fiber for dispersion compensation," IEEE Photonics Technology Letters, vol. 16, no. 6, pp. 1516-1518, 2004.

[12] F. Gérôme, J.-L. Auguste, and J.-M. Blondy, "Design of dispersion-compensating fibers based on a dual-concentriccore photonic crystal fiber," Optics Letters, vol. 29, no. 23, pp. 2725-2727, 2004.

[13] S. Yang, Y. Zhang, X. Peng et al., "Theoretical study and experimental fabrication of high negative dispersion photonic crystal fiber with large area mode field," Optics Express, vol. 14, no. 7, pp. 3015-3023, 2006.

[14] X. Zhao, G. Zhou, L. Shuguang et al., "Photonic crystal fiber for dispersion compensation," Applied Optics, vol. 47, no. 28, pp. 5190-5196, 2008.

[15] S. Kim, C.-S. Kee, D.-K. Ko, J. Lee, and K. Oh, "A dualconcentric-core photonic crystal fiber for broadband dispersion compensation," Journal of the Korean Physical Society, vol. 49, no. 4, pp. 1434-1437, 2006. 
[16] F. Poli, M. Foroni, M. Bottacini et al., "Single-mode regime of square-lattice photonic crystal fibers," Journal of the Optical Society of America A: Optics and Image Science, and Vision, vol. 22, no. 8, pp. 1655-1661, 2005.

[17] A. H. Bouk, A. Cucinotta, F. Poli, and S. Selleri, "Dispersion properties of square-lattice photonic crystal fibers," Optics Express, vol. 12, no. 5, pp. 941-946, 2004.

[18] P. S. J. Russell, E. Marin, A. Díez, S. Guenneau, and A. B. Movchan, "Sonic band gaps in PCF preforms: enhancing the interaction of sound and light," Optics Express, vol. 11, no. 20, pp. 2555-2560, 2003.

[19] M. K. Lee, P. S. Ma, I. K. Lee, H. W. Kim, and Y. Y. Kim, "Negative refraction experiments with guided shear-horizontal waves in thin phononic crystal plates," Applied Physics Letters, vol. 98, no. 1, Article ID 011909, 2011.

[20] G. P. Agrawal, Nonlinear Fiber Optics, Optics and Photonics, Academic Press, San Diego, Calif, USA, 4th edition, 2007.

[21] CUDOS, "MOF utilities," http://sydney.edu.au/science/physics/ cudos/research/mofsoftware.shtml.

[22] T. P. White, B. T. Kuhlmey, R. C. McPhedran et al., "Multipole method for microstructured optical fibers. I. Formulation," Journal of the Optical Society of America B: Optical Physics, vol. 19, no. 10, pp. 2322-2330, 2002.

[23] B. T. Kuhlmey, T. P. White, G. Renversez et al., "Multipole method for microstructured optical fibers. II. Implementation and results," Journal of the Optical Society of America B: Optical Physics, vol. 19, no. 10, pp. 2331-2340, 2002.

[24] “Corning SMF-28 CPC6 single-mode optical fibre," Product Information, Corning, Ithaca, NY, USA, 1998. 

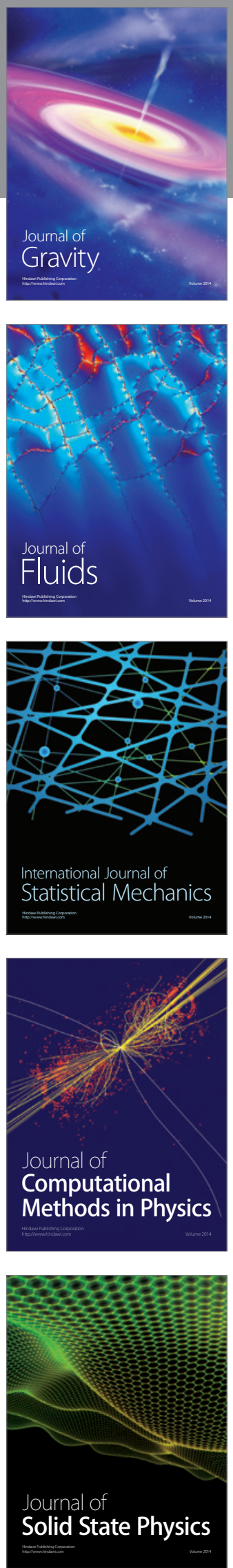

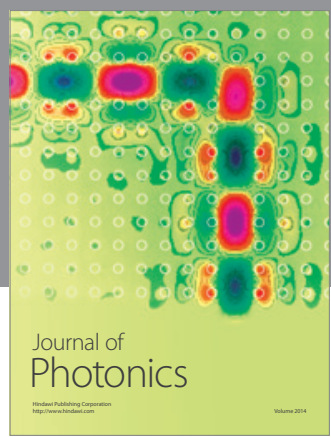

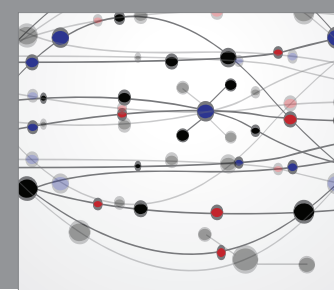

The Scientific World Journal

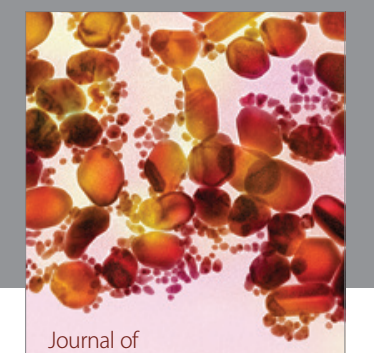

Soft Matter
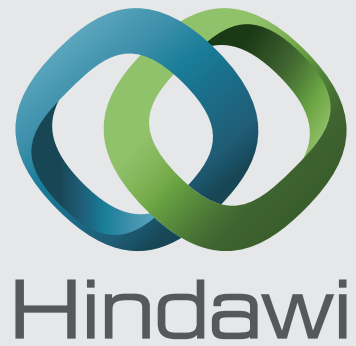

Submit your manuscripts at

http://www.hindawi.com
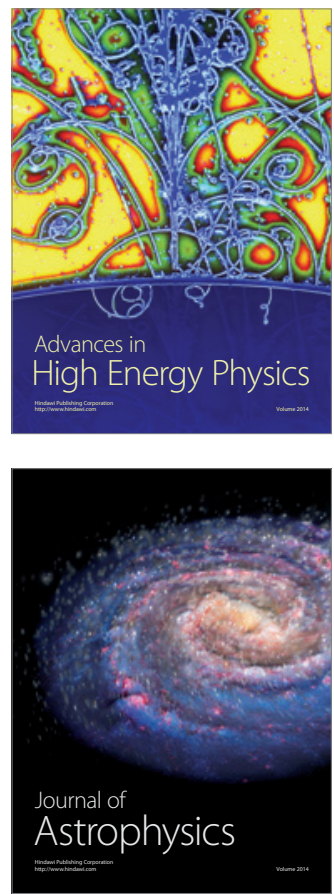
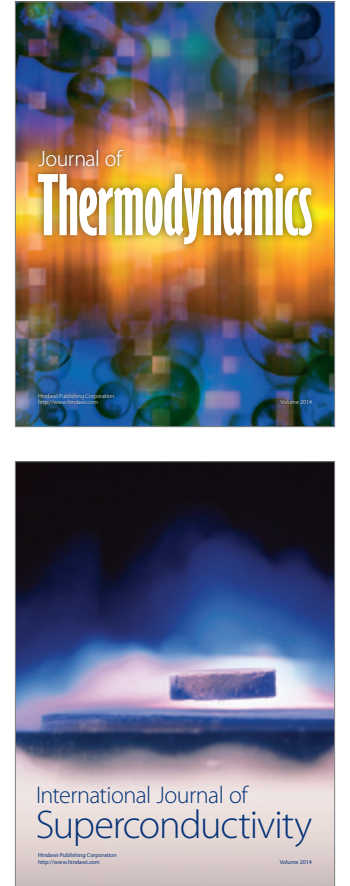
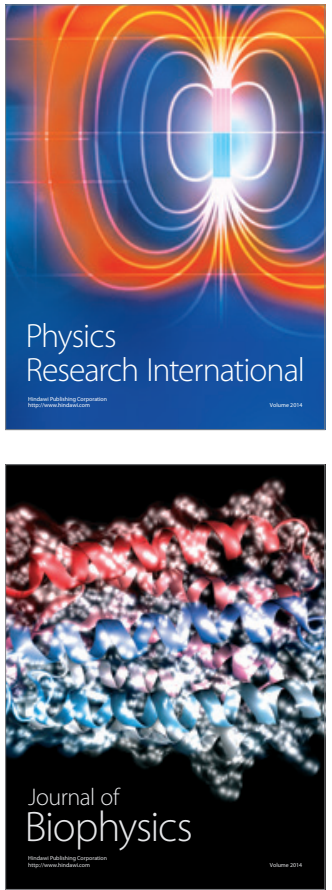
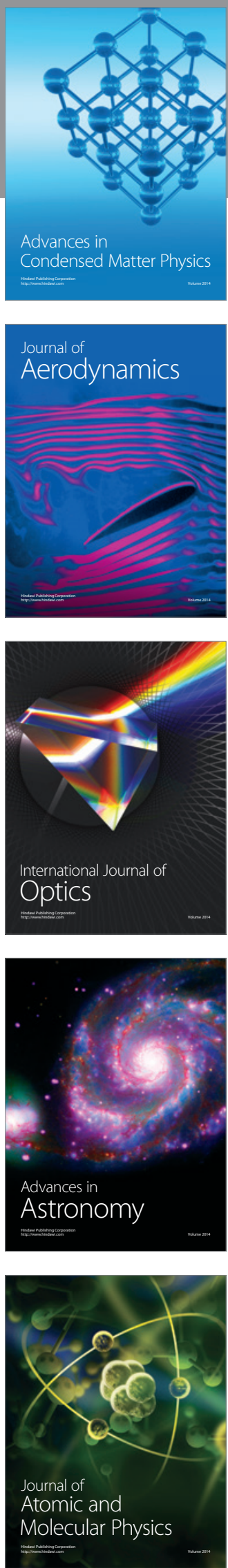